\title{
Frontières
}

\section{Le rapport aux traitements médicamenteux chez des personnes atteintes d'un cancer incurable}

\section{Suzanne Mongeau, Serge Daneault, Véronique Lussier et Louise Yelle}

Volume 16, numéro 1, automne 2003

Remède ou poison ?

URI : https://id.erudit.org/iderudit/1073762ar

DOI : https://doi.org/10.7202/1073762ar

Aller au sommaire du numéro

Éditeur(s)

Université du Québec à Montréal

ISSN

1180-3479 (imprimé)

1916-0976 (numérique)

Découvrir la revue

Citer cet article

Mongeau, S., Daneault, S., Lussier, V. \& Yelle, L. (2003). Le rapport aux traitements médicamenteux chez des personnes atteintes d'un cancer incurable. Frontières, 16(1), 57-63. https://doi.org/10.7202/1073762ar
Résumé de l'article

À notre époque, l'expérience pharmacologique de la maladie cancéreuse est devenue d'une grande complexité. Cette étude poursuit comme objectif de mieux connaître et comprendre le rapport que des personnes affligées d'un cancer incurable entretiennent à l'égard des traitements médicamenteux. Un devis qualitatif faisant appel à une analyse de contenu par théorisation a été retenu. L'analyse de l'ensemble des récits révèle que le rapport développé par les sujets rencontrés à l'égard des traitements médicamenteux s'inscrit dans une logique de combat. Tout au long de la maladie, les sujets adoptent deux postures différentes à l'égard des médicaments. En guise de conclusion, des questions en regard de la structure des services offerts et en regard de l’intervention sont soulevées. 


\section{Résumé}

À notre époque, l'expérience pharmacologique de la maladie cancéreuse est devenue d'une grande complexité. Cette étude poursuit comme objectif de mieux connaître et comprendre le rapport que des personnes affligées d'un cancer incurable entretiennent à l'égard des traitements médicamenteux. Un devis qualitatif faisant appel à une analyse de contenu par théorisation a été retenu. L'analyse de l'ensemble des récits révèle que le rapport développé par les sujets rencontrés à l'égard des traitements médicamenteux s'inscrit dans une logique de combat. Tout au long de la maladie, les sujets adoptent deux postures différentes à l'égard des médicaments. En guise de conclusion, des questions en regard de la structure des services offerts et en regard de l'intervention sont soulevées.

Mots clés : cancer incurable - traitements médicamenteux - combatintervention.

\section{Abstract}

This paper pursues an objective to better know and understand the relationship that exists between individuals afflicted with an incurable cancer and the medications offered as treatment. A qualitative research guideline based on the relevant literature has been selected. An analysis of an ensemble of personal narratives suggests that the relationship developed by the patients interviewed with the medication treatments is marked by a discourse of battle. Throughout the illness, patients adopt two different positions with respect to medications. To conclude, questions are raised with respect to the structure of the intervention services offered.

Key words: terminal cancer - medication treatments - battle-intervention.

$\begin{array}{lllllllll}\text { R } & \text { E } & \text { C } & \text { H } & \text { E } & \text { R } & \text { C } & H & \text { E }\end{array}$

\section{LE RAPPORT AUX TRAITEMENTS MÉDICAMENTEUX chez des personnes atteintes d'un cancer incurable}

\author{
Suzanne Mongeau, Ph.D., \\ Travail social, UQÀM. \\ $\overline{\text { Serge Daneault, M.D., Ph.D., }}$ \\ Hôpital Notre-Dame, CHUM. \\ Véronique Lussier, Ph.D., \\ psychologie, UQȦM. \\ Louise Yelle, M.D., \\ FRCP, hémato-oncologue, \\ Hôpital Notre-Dame, CHUM.
}

\section{MISE EN CONTEXTE}

Les progrès de la biomédecine au cours des dernières décennies ont fait en sorte que de nombreuses avancées ont été accomplies dans le traitement pharmacologique de la maladie cancéreuse. Ces progrès concernent, d'une part, le groupe des médicaments servant à éradiquer le cancer, à prolonger la vie ou à améliorer sa qualité, comme c'est le cas avec la chimiothérapie, et, d'autre part, le groupe des médicaments de soins de confort servant à soulager la douleur et les autres symptômes (nausée, vomissements, fatigue) pouvant être engendrés par certains traitements ou par la maladie elle-même. À notre époque, l'expérience pharmacologique de la maladie cancéreuse est devenue pour les personnes malades et pour les soignants d'une grande complexité. En effet, on retrouve une panoplie de médicaments qui peuvent être prescrits selon diverses combinaisons et qui peuvent être administrés par la bouche, par voies souscutanées, intramusculaires ou intraveineuses.

En lien avec ces nombreuses avancées au plan pharmacologique, plusieurs études se sont penchées sur la problématique des traitements médicamenteux mais en l'abordant, cette fois-ci, sous un angle psychologique et socioculturel. Jusqu'à maintenant, plusieurs aspects concernant, soit les expériences reliées à la chimiothérapie, soit les expériences reliées au soulagement de la douleur et des autres symptômes ont donné lieu à des recherches. En ce qui a trait au soulagement de la douleur, de nombreuses études ont cherché à identifier les différents facteurs pouvant expliquer que, malgré les avancées importantes faites dans ce champ, il demeure un pourcentage important de personnes souffrantes (Weiss et al., 2001; Fazeny et al., 2000). À cet égard, la littérature suggère que cette situation serait la résultante d'un ensemble de facteurs telles de fausses conceptions sur les médicaments antalgiques, que ce soit du côté du 
prescripteur ou des patients eux-mêmes, un manque de communication entre les personnes malades et le personnel soignant et, enfin, des lacunes dans les méthodes d'évaluation de la douleur (Ersek et al., 1999; Kimberley et al., 1999). Plus précisément, à partir du point de vue des personnes atteintes, Ward et ses collègues (1993, 1998) ont identifié huit obstacles au soulagement de la douleur : 1) la peur de l'accoutumance, 2) les préoccupations au sujet de la tolérance, 3) les préoccupations au sujet des effets secondaires, 4) le fatalisme (la douleur fait partie de l'expérience du cancer ; elle est inévitable), 5) le désir d'être un bon patient (les bons patients ne se plaignent pas ; cela ennuie les médecins), 6) la peur de distraire le médecin de la tâche de guérir le cancer, 7) la préoccupation selon laquelle une douleur qui s'accroît signifie que le cancer progresse, 8) la peur des injections.

Relativement à la chimiothérapie, certaines études se sont penchées sur l'expérience des personnes en traitement en l'abordant selon le prisme de la qualité de vie. Dans cette optique, la pertinence des traitements a été examinée en considérant les risques et bénéfices de ceux-ci (Elit et al., 2003 ; Simes et Coats, 2001 ; Jansen et al., 2001 ; Silvestri et al., 1998). Les regrets des personnes atteintes en regard de leur choix de traitement ont également été explorés (Clark et al., 2001). Les résultats de cette étude établissent une corrélation entre les regrets ressentis et le processus décisionnel ayant prévalu lors du choix de traitement. Les recherches qui ont étudié les attentes des personnes soumises à des traitements de chimiothérapie ont quant à elles bien mis en relief à quel point ces traitements sont investis d'un espoir de guérison. L'étude de Slevin et ses collaborateurs (1990) révèle que la majorité des personnes à qui des traitements de chimiothérapie ont été proposés ont affirmé qu'elles accepteraient de suivre les traitements, même s'il y avait seulement un pour cent de chance de guérison. Dans le même sens, pour ce qui est de la chimiothérapie palliative, l'étude de Doyle et al. (2001) révèle que, même s'il a été expliqué très clairement aux femmes atteintes d'un cancer de l'ovaire que les traitements avaient un but uniquement palliatif, un pourcentage important de participantes $(42 \%)$ pensait que les traitements allaient probablement les guérir. D'autres études ont exploré plus spécifiquement les souffrances engendrées par les effets secondaires des traitements comme, par exemple, la perte des cheveux et la fatigue (Kallich et al., 2002 ; Richer et Ezer, 2002 ; Morrow et al., 2001 ; Williams et al., 1999).

Mentionnons que l'on retrouve aussi un nombre significatif d'études qui ont cherché à documenter la question de l'informa-

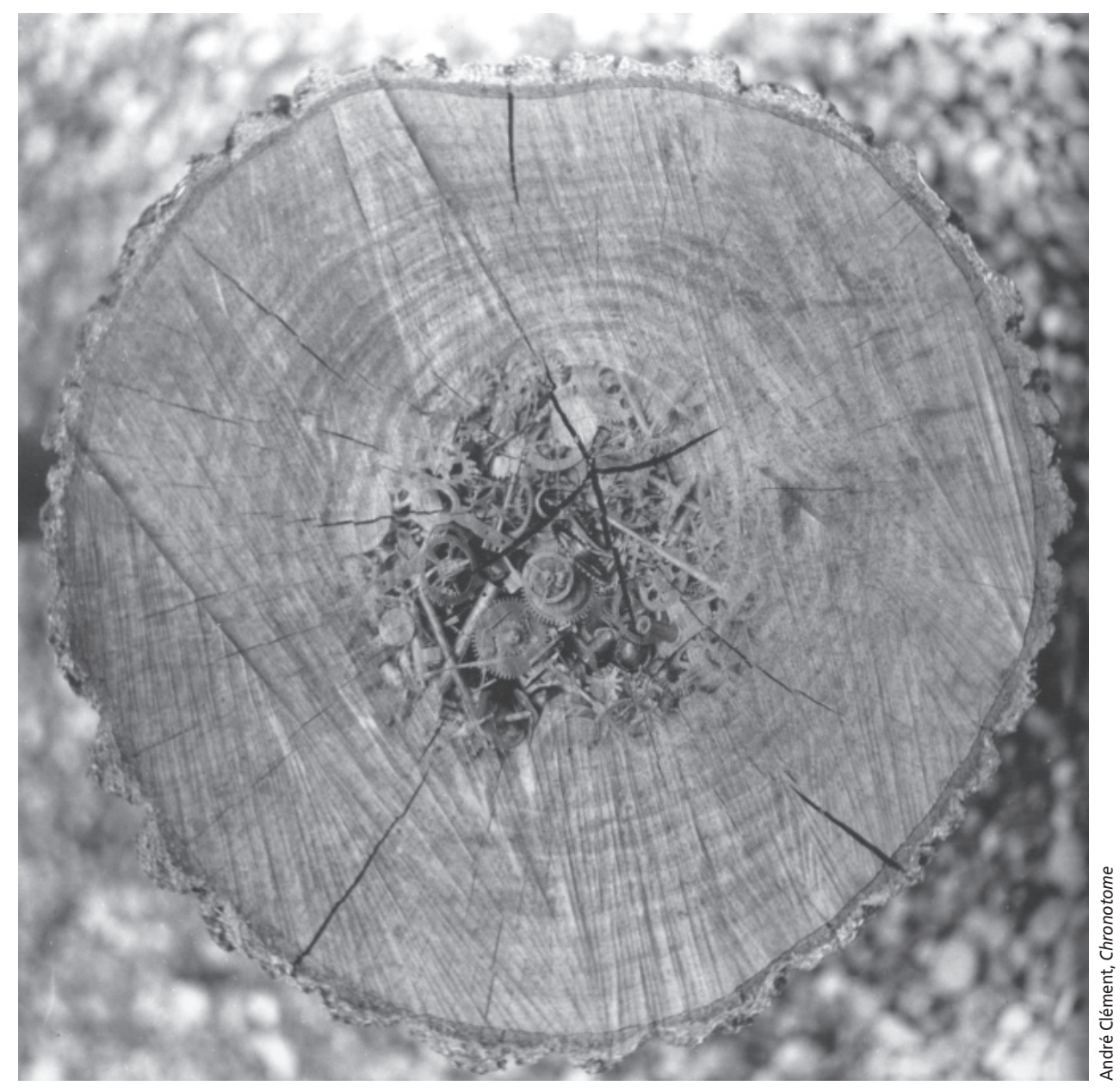

tion à l'égard de la maladie et des traitements reçus (Mills et Sullivan, 1999). Certaines d'entre elles l'ont abordée en essayant de connaître le niveau de satisfaction des personnes atteintes quant à l'information reçue (Uitterhoeve et al., 2003 ; Elf et Wikblad, 2001). On retrouve toutefois un ensemble de recherches provenant du domaine médical et du domaine de l'anthropologie qui ont étudié cette vaste et complexe question de l'information en l'englobant dans le spectre de la relation soignant-soigné et dans celui du rapport entretenu par les sujets à l'égard de leur maladie et de la mort. L'étude de Leydon et al. (2000) a exploré les différentes raisons qui pourraient expliquer pourquoi, à différents moments de leur maladie, certaines personnes atteintes de cancer s'abstiennent de rechercher des informations sur leur maladie et son évolution. La confiance que certaines personnes atteintes accordent à leur médecin ressort comme un motif pour s'abstenir de demander des informations supplémentaires. Pour ces patients, le médecin représente l'expert qui détient le savoir. Dans cette perspective, ces mêmes patients craignent de transgresser leur rôle de malade et préfèrent être de bons patients. Le besoin de préserver un espoir est évoqué comme un autre motif pour s'abstenir d'aller chercher des informations. Enfin, des personnes malades s'empêchent de demander des informations supplémentaires, car elles considèrent que le médecin manque de temps et que d'autres malades peuvent avoir des besoins plus importants que les leurs.

L'étude de The et al. (2000), de même que celle de DelVecchio Good et al. (1993) ont mis en lumière les diverses stratégies utilisées par les personnes malades et par les prescripteurs pour garder à distance certaines informations. The et al. (2000) démontrent la collusion qui prévaut entre le médecin et la personne malade pour maintenir un optimisme trompeur à l'égard de la maladie et des traitements. Dans les interactions, l'accent est mis sur la technicité des traitements, ce qui confirme d'ailleurs ce que d'autres études avaient aussi relevé (Uitterhoeve et al., 2003 ; Ford et al., 1996). L'étude de DelVecchio Good et al. (1993) a bien mis en relief le fait que les oncologues se voient confier le mandat culturel de maintenir l'espoir chez les patients, ce que celle de Saillant (1988) avait déjà mis en évidence. Pour remplir ce mandat, ils utilisent diverses stratégies. Pour éviter d'aborder le dénouement possible de la maladie, les échanges entre médecins et soignés sont axés sur l'immédiateté, le calendrier des traitements, les effets secondaires possibles et sur la vie quotidienne. 


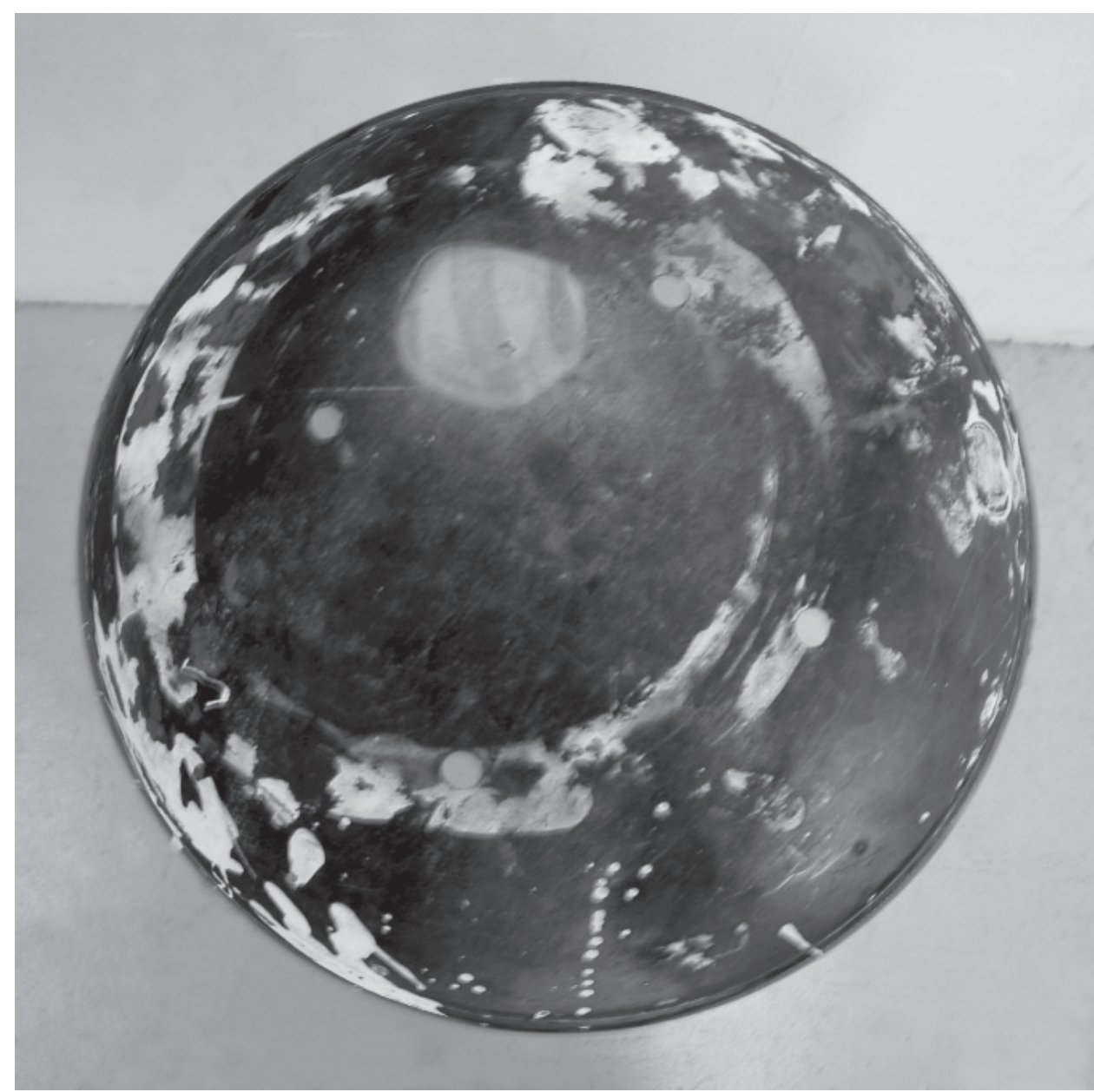

Ce bref regard sur la littérature existante dans le domaine des traitements médicamenteux pour des personnes atteintes d'un cancer nous permet de constater que les connaissances acquises, bien que significatives, sont morcelées. Les diverses études menées se sont penchées soit sur l'expérience des sujets en regard des médicaments pouvant servir à éradiquer le cancer ou à prolonger à tout le moins la vie, soit sur les médicaments de confort. Jusqu'à maintenant, aucune étude ne s'est penchée sur le rapport que les personnes affligées d'un cancer incurable entretiennent à l'égard de l'ensemble des traitements médicamenteux, et ce depuis l'obtention du diagnostic d'incurabilité. La présente étude cherche à pallier ce manque de connaissances en poursuivant comme objectifs de mieux connaître et comprendre ce rapport et d'identifier les principales caractéristiques qui s'en dégagent.

\section{MÉTHODOLOGIE}

Les résultats présentés ici proviennent de témoignages recueillis dans le cadre d'une étude plus vaste ${ }^{1}$ menée à Montréal et portant sur la souffrance des personnes atteintes d'un cancer incurable. Compte tenu de la complexité du phénomène à l'étude, un devis qualitatif faisant appel à une analyse de contenu par théorisation types de cancers reflétant potentiellement diverses expériences avec les traitements reçus sont représentés dans l'échantillon: poumon, sein, foie, ovaire, colon, cerveau, gorge, système hématopoïétique, œsophage, pancréas, voies biliaires et mélanome. Enfin, les sujets provenaient de toutes les classes sociales.

Les données ont été recueillies à partir de trois sources: 1) des entrevues individuelles semi-dirigées audio-enregistrées effectuées par quatre cochercheurs, 2) des notes de terrain prises par les interviewers, 3) les dossiers médicaux auxquels les sujets avaient donné accès aux chercheurs par le formulaire de consentement. L'analyse a été effectuée à partir des transcriptions intégrales de tout le matériel recueilli. Ce matériel a été codifié, catégorisé et analysé en vue de définir des thématiques émergentes. Celles-ci ont été soumises à l'accord « interjuges » de trois des cochercheurs provenant de quatre disciplines différentes (médecine, anthropologie, psychologie, travail social), jusqu'à l'obtention du consensus et saturation de l'information. Pour les fins de cet article, seules les catégories ayant trait aux traitements médicamenteux ont été retenues, traitées et analysées. Elles ont, par la suite, été mises en relation afin que nous puissions obtenir une compréhension approfondie du rapport que les sujets entretiennent à l'égard de l'ensemble des traitements médicamenteux. La modélisation s'est construite en établissant des liens entre les différents éléments constituant le phénomène étudié. Celle-ci a été mise à l'épreuve par la recherche de cas négatifs et par une validation des résultats, auprès d'une partie de l'échantillon qui a participé à une deuxième entrevue $(\mathrm{N}=5)$. Toutes les analyses ont été effectuées à l'aide du logiciel QSR NUD.IST ${ }^{\circledR}$.

\section{LA DYNAMIQUE RELATIONNELLE SOIGNANT-SOIGNÉ}

Comme nous l'avons souligné précédemment, les résultats présentés dans cet article proviennent d'une étude plus vaste poursuivant comme objectif de mieux comprendre la souffrance des personnes atteintes d'un cancer incurable, et ce en étudiant le rôle, sur ces souffrances, des interventions effectuées par les soignants. À cet égard, les données recueillies ont permis de mettre en lumière une dynamique propre à la relation soignant-soigné, que l'on peut caractériser par un évitement de la souffrance. Comme chez les sujets rencontrés, cette dynamique particulière constitue la toile de fond sur laquelle se construit le rapport aux traitements médicamenteux, il nous a semblé important de nous y attarder avant de considérer les résultats portant spécifiquement sur les traitements médicamenteux. 
Le soignant, en tant qu'acteur et représentant du système institutionnel de santé, et le soigné sont engagés ensemble dans une série d'interactions basées sur un évitement de la souffrance qui s'opère de manière circulaire. Malgré l'évidente inégalité du rapport de force en présence (le soignant et le système au sein duquel il agit détiennent un pouvoir plus important que celui que possède le soigné), on peut observer une certaine forme d'accord implicite entre les deux parties quant à maintenir à distance la souffrance. De part et d'autre, il s'agit d'abord de ne pas ouvrir les digues de la souffrance, de ne pas donner prise au chaos et au désespoir que la maladie grave peut engendrer. Tout ce qui risque d'échapper au contrôle de soi est considéré comme une menace.

$\mathrm{Au}$ sein du système institutionnel, les interactions entre soignants et soignés s'opèrent dans un contexte qui décourage l'expression de la souffrance. Il suffit de penser à l'achalandage des unités de soins et à la surcharge de travail du personnel. À cet égard, une femme qui avouait être pleine d'inquiétude et d'appréhension nous indiquait comment elle avait fait face à ce contexte: "J'ai pas commencé à y parler de ça. Pas grand temps... J'ai dix minutes avec elle. Pis c'est plein dans la salle d'attente. Non, elle ne me connaît pas» (Louise). Il semble donc que les personnes souffrantes doivent se résoudre à taire beaucoup de choses. Les sujets rencontrés ont aussi mentionné la rigidité du système ; pour eux, il est risqué de vivre le doute, de porter un regard critique sur les soins reçus, de poser des questions, d'insister. Les personnes atteintes ressentent l'importance, voire la nécessité de ne pas s'aliéner le personnel soignant si elles veulent être en mesure de vaincre le cancer. Les demandes que les sujets perçoivent du système, et auxquelles dans la très grande majorité des cas ils obéissent, sont en fait des demandes propres à la culture ambiante : on doit être courageux, stoïque et positif. Au cœur de cet enchaînement d'interactions, la souffrance est à l'étroit. Elle doit prendre des proportions raisonnables, être contenue et se vivre sur le mode de la sagesse. En quête de structure et de remparts contre la mort, les sujets malades vont ainsi se plier à ces contraintes et s'adapter à l'étroitesse de cette zone car leurs propres besoins cadrent avec les exigences du système. Où il y aurait lieu de s'attendre à une évolution plus souple vers l'issue fatale de la maladie, on observe une certaine forme d'enlisement dans la collusion. En somme, de leur côté les soignants pressentent et respectent en quelque sorte le besoin des sujets de se prémunir contre un risque de débordement d'expression de la souffrance et de leur côté les sujets, perçevant la réticence des soignants et du système à ouvrir les digues, en viennent, à leur tour, à protéger le système.

Or cet enlisement dans la collusion ne peut durer qu'un temps puisque les personnes atteintes vivent avec un cancer déclaré incurable. C'est ainsi que devant le constat inéluctable de l'échec curatif, les personnes malades ressentent une brusque dissolution du pacte conclu avec les soignants car ils ne peuvent plus lutter côte à côte. Les sujets rencontrés ressentent alors une certaine forme de désolidarisation face à l'imminence de la mort, maintenant incontournable. À cette étape et dans un premier temps, le transfert aux soins palliatifs symbolise le terme d'un parcours fait d'évitements. Les soins palliatifs écopent, en quelque sorte, de ce report de la souffrance. Pour les soignés qui, par la suite, sont réceptifs à l'ouverture des soins palliatifs pour ce qui est d'un lâcher prise à l'égard de la souffrance, il y a une certaine amertume à voir celle-ci accueillie seulement en fin de parcours. Paradoxalement, la dynamique relationnelle d'évitement de la souffrance qui a prévalu entre soignants et soignés jusqu'à l'étape ultime produit exactement ce qu'elle cherchait à éviter ; une souffrance qui s'additionne à celle que le seul fait de la maladie implique. Dans la présentation des résultats qui suivent, nous verrons comment cette dynamique participe des rapports que les sujets entretiennent avec les traitements médicamenteux.

\section{RÉSULTATS}

L'analyse de l'ensemble des récits révèle que le rapport développé par les sujets rencontrés à l'égard des traitements médicamenteux s'inscrit dans une logique bien spécifique : celle du combat. Un combat mené, bien sûr, contre la maladie grave, mais aussi et surtout contre la mort. Selon l'évolution de la maladie, mais toujours dans la logique du combat, les médicaments sont perçus tantôt comme des armes puissantes, tantôt comme des adversaires redoutables, tantôt comme des alliés qui peuvent apporter un répit temporaire. Bien que les sujets aient fait référence dans leur discours à différents médicaments, c'est principalement autour des traitements de chimiothérapie que s'organise le rapport aux traitements médicamenteux.

Après l'annonce du diagnostic où une frayeur extrême a été ressentie à l'égard de la mort, tous les sujets rencontrés se sont mobilisés rapidement pour mettre en branle le combat. À cette étape, la chimiothérapie, même si elle n'a que des visées palliatives, est perçue comme l'arme la plus efficace pour mener la bataille. Les médicaments antalgiques et ceux qui sont administrés pour soulager les différents inconforts (nausées, vomissements, fatigue) sont alors placés à l'arrière-scène. L'annonce de la fin des traitements de chimiothérapie vient signer la défaite. Les sujets ressentent alors beaucoup d'amertume envers les traitements de chimiothérapie et les effets toxiques qu'ils ont engendrés. Avec l'annonce de la fin des traitements, les sujets sont alors confrontés en direct avec la mort. Au niveau des pratiques d'intervention, l'annonce de la fin des traitements est en général couplée avec l'offre d'un transfert à l'équipe des soins palliatifs. Dans un premier temps, ce transfert est perçu négativement. Par la suite, après avoir eu des contacts avec les soignants des soins palliatifs, l'ensemble du discours des sujets est orienté vers l'importance d'une mort paisible et sans douleur.

Tout au long de la trajectoire de leur maladie, les combattants adoptent donc deux postures différentes à l'égard des médicaments. Pendant les traitements de chimiothérapie, ils prennent une position de guerrier qui garde l'espoir de guérir. Après l'annonce de la fin des traitements, leur imposant un changement de rythme brutal, ils adoptent une position de vaincu. Le rapport aux médicaments développé pendant le combat, de même que celui développé après la défaite sont maintenant décrits et analysés dans ce qui suit.

\section{PENDANT LE COMBAT}

Comme nous l'avons déjà souligné, pour contrer l'effroi qui les a submergés lors du diagnostic de l'incurabilité, tous les sujets rencontrés se sont mobilisés rapidement et se sont engagés avec énergie dans un combat contre la maladie et la mort: « [...] là, j'ai piqué ma crise dans l'bureau du médecin, pis après, je me suis dit je suis capable de me battre, je suis capable de passer par-dessus. Pis je me suis toujours battue»(Lynne $)^{2}$. Dans cette logique du combat, les traitements de chimiothérapie sont alors fortement investis. Ils signent, bien sûr, la gravité de la maladie, mais ils incarnent surtout un espoir de guérison, et ce, même si tous les sujets ont été avisés du caractère incurable de leur cancer :

Ah! Au début, je me disais si... si j'ai des traitements de chimio, ça veut dire que c'est quasiment la fin, t'sais [...] Au début, j'ai pensé ça, après ça, je me suis dit, il y en a beaucoup qui sont... qui ont passé au travers ça fait que je me suis dit, ben, j'vas essayer. Pis j'ai continué à avoir les traitements.

(Adèle)

Dans leur état de grande vulnérabilité, les personnes malades ont besoin de protection et tous les médicaments prescrits visant à vaincre le cancer sont perçus, dans ce contexte, comme des armures qui les 
DEVANT LE CONSTAT INÉLUCTABLE DE L'ÉCHEC CURATIF, LES PERSONNES MALADES RESSENTENT UNE BRUSQUE DISSOLUTION DU PACTE CONCLU AVEC LES SOIGNANTS CAR ILS NE PEUVENT PLUS LUTTER CÔTE À CÔTE.

préservent de l'ennemi. Ils sont en quelque sorte des remparts contre la mort comme en témoigne Solange :

Pis après ça, au fur et à mesure que tu lis pis qu'il y a de la chimio pis tout ça, toute l'évolution, ça fait que ça devient moins noir au bout, là. Parce qu'au début, là, c'est un tableau noir, là. Tu vois pas rien en avant, là. Il faut qu'il s'éclaircisse ce tableau-là.

De plus, les traitements de chimiothérapie semblent contrer, chez certains, l'effet de chaos et de désordre créé par la maladie et par la peur de mourir. Ils constituent en ce sens un repère structurant, ne serait-ce que par le rythme que la prise de ceux-ci impose. Paradoxalement, ils introduisent une certaine continuité dans cette expérience de rupture que constitue la maladie grave. Par ailleurs, chez certains, la prise de médicaments pouvant éradiquer le cancer mais engendrant des effets secondaires importants leur rappelle la maladie qu'ils essaient d'oublier : "Tu passes devant le miroir pis tu te vois, pas un poil sur la tête, tu te vois amaigrie ou enflée, moi, c'est la maladie que je vois. » (Françoise)

Plusieurs récits dévoilent que les personnes atteintes sont prêtes "presqu'à tout» pour rester en vie. Certaines sont parfois prêtes à payer des coûts astronomiques pour des médicaments, si cela peut les sauver de la mort. D'autres partent à la recherche de la pilule ou du traitement miracle qui pourrait les guérir. Elles font des démarches pour trouver le meilleur médecin en ville, celui qui pourra leur offrir un traitement, alors qu'un autre les aura déjà avisées de la futilité d'entreprendre d'autres traitements. Dans leur logique, tout se passe comme si chaque médecin pouvait concocter sa propre potion magique :

[...] Ça fait que finalement une de mes amies a trouvé des recherches, elle a trouvé des chercheurs à Toronto pis en Ontario, pis des recherches sur le

Vincristine pour les tumeurs au cerveau et elle dit c'est à Notre-Dame, c'est le Docteur G. Ça fait qu'on a fait les démarches. (Sophie)

Même si les traitements de chimiothérapie les rendent souvent très malades (nausées, vomissements, fatigue) et transforment leurs corps (chute des cheveux, gonflements), plusieurs éléments de leur discours révèlent qu'à cette étape, ils sont prêts à « en endurer », car comme le disait un des sujets, ils veulent guérir: «[...] Là j'ai dit: Mon Dieu! Donnez-moi des souffrances, donnez-moi des douleurs, mais si vous gardez la vie, j'accepte» (Berthe). Le chemin vers la guérison est donc perçu, comme l'avait déjà souligné Chicaud (1998), comme un combat de type sacrificiel. Pour certains, tout se passe comme s'il fallait encore souffrir pour guérir. Pendant le traitement, la majorité exprime donc peu d'agressivité envers les effets secondaires, qui les rendent bien souvent plus malades que la maladie elle-même. Les témoignages laissent entendre qu'ils craignent de réactiver leur ennemi en exprimant leur agressivité. «[...] Ça m'étouffe, ça me gonfle jusque-là ces médicaments-là, mais ils sont efficaces pour la tumeur, ça fait que là, là-dessus, ça l'emporte. » (Sophie)

Pendant le combat, l'incertitude, les doutes, l'ambivalence quant à l'efficacité des traitements est rarement abordée directement. Les sujets y font parfois allusion, mais seulement en effleurant l'idée. À cette période, certains semblent même éprouver des difficultés à prononcer le mot morphine, comme si ce médicament était associé de trop près à la mort. Les sujets semblent avoir oublié que les traitements de chimiothérapie n'ont qu'une visée palliative. Pendant le combat, l'atmosphère qui semble régner entre les prescripteurs et les personnes malades s'apparente à ce que The et al. (2000) ont appelé «l'aura curative». Il y a toujours, sous-entendu, un espoir de triompher du mal, de faire mentir les statistiques, d'être l'exception, le miracle. Le prolongement de la vie par les traitements de chimiothérapie, même quand il s'agit «clairement» d'un simple report d'échéance ou d'une amélioration de la qualité de vie à court terme, finit par ressembler à une promesse d'immortalité : «[...] Je me suis dit qui fallait pas que je m'arrête à ça. J'ai dit j'vas défier les statistiques [...] J'ai dit, il aura pas ma peau comme ça, lui, là. » (Solange)

Pendant le combat, la relation avec le prescripteur est fortement investie. La parole du médecin revêt en effet une très grande importance. On retrouve toutefois deux postures différentes à l'égard du prescripteur. Pour rester en vie, certains sont prêts à prendre une position d'objet face à la médecine. Ils ont alors besoin de croire en leur médecin. Ils mettent en lui une confiance absolue, aveugle et inébranlable. Ils idéalisent la médecine à travers le médecin. Ils investissent leur médecin d'un pouvoir surhumain et le perçoivent comme un être capable d'exterminer leur ennemi, comme l'exprime Florence : « T'sais, bon, t'as un cancer, on va essayer ça, c'est supposé marcher. Si le docteur te dit, c'est supposé marcher, c'est parce que ça va marcher. » D'autres veulent toutefois garder un statut de sujet même s'ils s'inscrivent dans une logique de combat. Ils veulent garder une position active dans les traitements. Ils ont besoin de se faire reconnaître comme personne et ils refusent une relation d'infantilisation. Ils veulent rester acteur de leur propre vie.

[...] J'ai dit, moi, je veux pas de quelqu'un qui contrôle ma vie en entier pis qui décide de tout, pis que je n'ai jamais, jamais, mon mot à dire. Je regrette, mais moi je ne fais pas partie de ces patientes-là [...] qui se laissent faire. [...] J'veux bien faire confiance, mais faire confiance et fermer les yeux et pas savoir dans quoi on s'embarque c'est pus faire confiance, là. C'est comme quasiment de l'aveuglement. Alors, moi, je me suis sentie, comme une enfant, traitée comme une enfant. Vraiment, l'infantilisation... Tu sais plus ce que tu veux, tu sais plus ce que t'es, tu sais plus si tu as le droit de parole [...] (Céline)

Pour les personnes de ce groupe, il est important que les prescripteurs reconnaissent qu'elles possèdent une expertise spécifique liée au fait que ce sont elles qui prennent les médicaments et qui en ressentent les effets. Dans leur logique, elles sont les mieux placées pour décider d'arrêter de prendre un médicament qui engendre trop d'inconforts. Toutefois, même celles qui veulent être reconnues comme sujets et partenaires ne souhaitent pas se faire trop directement rappeler par leur médecin la visée palliative des traitements. Leur position à l'égard de l'information ne s'inscrit pas dans une logique binaire, celle de savoir ou ne pas savoir. Leurs besoins et leurs attentes sont plus complexes qu'il peut paraître. À cet effet, le discours de Céline traduit de manière éloquente le rapport ambigu que certaines personnes affligées d'un cancer incurable peuvent entretenir à l'égard de l'information. Ainsi, d'une part, elle soutient qu'elle désire savoir :

[...] me semble que c'est clair; pas besoin d'être psychologue pour comprendre que la personne que t'as 
devant toi, le patient ou la patiente que t'as devant toi elle est de celles ou de ceux qui veut savoir. [...] Elle ne veut pas s'en aller en ne sachant pas, pis en restant dans les limbes [...].

D'autre part, sa réaction devant le rappel du médecin quant à l'aspect palliatif du traitement met en relief son besoin de garder une distance avec le caractère incurable de son cancer:

[...] On me dit: «Ah! C'est beau, pour le moment, c'est stable. » [...] J'pense la prochaine fois que j'vais voir l'oncologue, j'vais y dire: «J'aimerais ça que vous marquiez un petit mot dans votre dossier, que vous puissiez bannir pour le moment. Gardez juste c'est beau et l'état est stable, oublions le pour le moment.» [...] Moi, je trouve que ce petit mot-là pour le moment, c'est l'image de l'épée de Damoclès : "Ma fille, profites-en parce que la rechute va arriver. »

Même si les sujets rencontrés adoptent des positions différentes à l'égard du prescripteur, tous ont exprimé, d'une façon ou d'une autre, le besoin qu'on s'intéresse à eux comme personne et ce, au-delà des aspects techniques des traitements. Ils n'ont donc pas toujours besoin d'en savoir davantage. Ils demandent plutôt un accompagnement et une présence de la part de quelqu'un qui soit en mesure de rentrer dans leur monde et dans leur logique.

\section{LA DÉFAITE... ET APRÈS}

Le rapport aux traitements médicamenteux se modifie lorsque les sujets sont placés, sans ambiguité, devant l'inutilité de la poursuite des traitements de chimiothérapie. Pour tous, la fin de ceux-ci vient signer la défaite. Plusieurs ont évoqué, d'une manière ou d'une autre, la forte impression de non-sens qui les a alors habités. À quoi sert d'avoir mis autant d'énergie dans la bataille et d'avoir subi tous ces effets secondaires si tout cela se termine par la défaite? $\mathrm{Au}$ cours de cette période, les sujets rencontrés se permettent plus facilement de libérer leur agressivité face aux divers traitements subis, et, d'autre part, par rapport à ceux qui les ont prescrits. Parallèlement au sentiment d'absurdité et d'injustice ressenti devant l'échec, plusieurs sujets mentionnent l'impuissance qu'ils ont alors ressentie: «J'avais... j'avais pas de munitions, j'avais rien, complètement démuni » (Benoît).

Plusieurs ont aussi fait référence au fait que leur oncologue avait utilisé dans des termes plus ou moins explicites la formule «On ne peut plus rien pour vous» afin de leur signifier l'inutilité de la poursuite des

POUR RESTER EN VIE, CERTAINS SONT PRÊTS À PRENDRE

UNE POSITION D'OBJET FACE À LA MÉDECINE.

traitements : «Ça m'a débiné au coton, j’ai eu l'impression que lui, ce qu'il se disait, c'est qu'il y avait pus rien à faire. On laisse aller, bon, on mettra les soins palliatifs. Lui, son impression, c'est qu'il y avait rien à faire pour moi » (Édouard). Pour les sujets rencontrés, cette formule a été perçue comme une condamnation à mort.

Par rapport au mode guerrier endossé par les sujets pendant les traitements de chimiothérapie, l'arrêt de ceux-ci impose l'adoption d'un changement de posture radical. Plusieurs ont décrit ce moment comme s'ils ne l'avaient pas vu venir. Pour eux, l'espoir a été brisé trop brusquement et de manière absolue. L'abandon de la lutte ne s'est donc pas vécu dans le cadre d'une transition progressive. De plus, comme l'annonce de la fin des traitements est souvent couplée avec l'offre de soins palliatifs, les sujets se sont sentis abandonnés. Ils doivent affronter une double perte: faire le deuil d'une guérison possible et perdre le médecin qui les avait suivis jusqu'alors.

Le passage à la sphère "où l'on ne se bat plus », associé à l'offre de soins palliatifs, prend bien sûr dans un premier temps une connotation négative. Certains, comme Solange, se sentent dévalorisés : "J'me suis dit: Débrouille-toi toute seule, ma fille [...] parce que là, j'suis plus un gros cas pour eux autres, dans l'fond. » D'autres, comme Sophie, perçoivent les soins palliatifs comme un mouroir :

Ah! Les soins palliatifs, pour moi, c'est tous des p'tits vieux, pis tu t'en vas mourir ben raide [...] ils te disent que tu peux mourir, tu peux partir [...] ils font ce qu'ils pensent bien faire, mais toi en tant que mourant, ça fait pas ton affaire, parce que tu veux pas mourir.

Bref, tout ce qui est palliatif se trouve dans un premier temps assimilé à l'abandon et à la mort.

La perception des sujets à l'égard des soins palliatifs et à l'égard des médicaments prescrits se modifie par la suite lorsqu'ils sont en contact avec l'équipe des soignants. Les soins palliatifs sont alors perçus non plus seulement comme un endroit où l'on va mourir, mais aussi comme un lieu où l'on peut recevoir des petits soins et des petites attentions: «[...] Pis tout l'monde est fin, pis tout l'monde est gentil, tout l'monde est prévenant»(Florence). Dans ce lieu et à cette étape de la maladie, le rapport aux médicaments antalgiques se modifie. Le soulagement de la douleur et des inconforts revêt alors une importance capitale, même si certains éprouvent encore des réticences par rapport à la morphine. En soins palliatifs, les personnes parlent de la douleur et de l'importance de son soulagement, alors qu'auparavant, pendant le combat, ils avaient accordé peu de place à cette dimension : «[...] C'est la première fois [à l'unité des soins palliatifs] qu'ils réussissent à contrôler ma douleur par les médicaments » (Mylène).

\section{QUESTIONNEMENTS ET IMPLICATIONS}

Les résultats recueillis dans cette étude soulèvent un certain nombre de questions en regard de la structure même des services offerts et en regard de l'accompagnement et de l'intervention. Nous avons mis en évidence comment les sujets, dans leur parcours, doivent faire face à un changement de rythme brutal à l'égard de la maladie et des significations accordées aux traitements médicamenteux. Lorsqu'ils reçoivent des traitements de chimiothérapie et qu'ils relèvent de l'équipe d'oncologie, les sujets adoptent la position d'un combattant qui cherche à guérir à tout prix et à repousser la mort dans ses plus lointains retranchements. À cette étape, ils perdent de vue la visée palliative des traitements, ce qui confirme certains résultats de l'étude de Doyle (2001). Avec l'annonce de la fin des traitements, les sujets sont habituellement pris en charge par l'équipe des soins palliatifs et ils sont encouragés à adopter une position de « lâcher prise » tout en espérant une mort paisible accompagnée d'un soulagement de la douleur adéquat. Or comme ce changement de rythme brutal semble difficile à assimiler et à digérer, on peut se demander s'il n'y aurait pas avantage à ce que le monde de l'oncologie et celui des soins palliatifs s'interpénètrent davantage, ce qui pourrait assouplir le passage du curatif au palliatif. Dans cette perspective, on peut penser que des changements devront être apportés à la structure même des services.

Nous avons également identifié la présence de deux tendances à l'intérieur de notre échantillon. Certaines personnes malades préfèrent mettre leur vie et leur corps entre les mains de la médecine alors que d'autres veulent jouer un rôle actif dans leur traitement. Pour les personnes du premier groupe, la notion de participation aux traitements ne semble donc pas faire de sens et pourrait même soulever des angoisses 
additionnelles si elle était présentée comme un impératif, alors que pour celles du deuxième groupe, il importe de reprendre un certain contrôle sur leur vie, que le cancer leur a enlevé, en adoptant une position active. Nous avons toutefois relevé que même les personnes qui veulent être reconnues comme sujets et partenaires à part entière entretiennent un rapport ambigu, voire paradoxal à l'égard de l'information. Elles veulent à la fois savoir et ne pas savoir. Au-delà de ces divergences, tous les sujets apprécient que les échanges avec les soignants ne se résument pas aux aspects techniques des traitements. Les résultats ont également mis en relief comment le rapport entretenu par les sujets à l'égard des traitements médicamenteux ne s'inscrit pas dans une logique rationnelle.

Devant ces différents constats, il convient de se demander comment, dans leurs pratiques d'intervention, les soignants peuvent prendre en considération la nature évolutive, paradoxale, idiosyncrasique et irrationnelle du rapport développé entre les personnes malades, leur maladie et les traitements médicamenteux subis. À cet effet, les sujets rencontrés ont fourni dans leurs récits quelques pistes de réponse. Ils souhaitent, bien sûr, une compétence scientifique, mais aussi un accompagnement. Ce dernier impliquerait un intérêt de la part du soignant pour l'univers du soigné, c'est-à-dire un espace d'écoute pour ses ambivalences, ses contradictions, et l'unicité de son expérience de la maladie. Bref, un accompagnement qui saurait médiatiser le rapport que les personnes malades entretiennent à l'égard de la mort et qui pourrait, par conséquent, assouplir le rapport aux traitements médicamenteux ${ }^{3}$.

\section{Bibliographie}

CHICAUD, M.-B. (1998). La crise de la maladie grave, Paris, Dunod.

CLARK, J., N. WRAY et C. ASHTON (2001). "Living with treatment decisions: Regrets and quality of life among men treated for metastatic prostate cancer », Journal of Clinical Oncology, $\mathrm{n}^{\circ}$ 19, p. 72-80.

DELVECCHIO GOOD, M.-J., T. MUNAKATA et Y. KOMAYASHI (1993). «Temps narratif et incertitude en médecine clinique », Anthropologie et Sociétés, vol. 17, nos 1-2, p. 79-98.

DOYLE, C., M. CRUMP, M. PINTILIE et A. OZA (2001). "Does palliative chemotherapy palliate? Evaluation of Expectations, outcomes, and costs in women receiving chemotherapy for advanced ovarian cancer", Journal of Clinical Oncology, $\mathrm{n}^{\circ}$ 19, p. 1226-1274.

ELF, M. et K. WIKBLAD (2001). «Satisfaction with information and quality of life in patients undergoing chemotherapy for cancer. The role of individual differences in infor- mation preferences », Cancer Nursing, n 24 , p. 351-356.

ELIT, L., C. CHARLES, I. GOLD, A. GAFNI, S. FARRELL, S. TEDFORD, D. DAL BELLO et T. WHELAN (2003). "Women's perceptions about treatment. Decision making for ovarian cancer», Gynecology Oncology, vol. 28, n ${ }^{\circ}$ 2, p. 89-95.

ERSEK, M., B. MILLER-KRAYBILL, A. DU PEN (1999). "Factors hindering patient's use of medications for cancer pain ", Cancer Practice, vol. 7, n 5, p. 226-232.

FAZENY, B., M. MUHM, I. HAUSER, C. WENZEL, P. MARES, A. VBERZLANOVICH, H. HAGMEISTER et C. MAROSI (2000). "Barriers in cancer pain management», The Middle European Journal of Medicine, p. 978-981.

FORD, S., L. FALLOWFIELD et S. LEWIS (1996). "Doctor-patient interactions in oncology ", Social Science and Medicine, vol. 42, n ${ }^{\circ} 11$, p. 1511-1519.

JANSEN, S., J. KIEVIT, M. NOOIJI et al. (2001). "Patients' preferences for adjuvant chemotherapy in early-stage breast cancer: Is treatment worthwile? ", British Journal of Cancer, $\mathrm{n}^{\circ} 84$, p. 1577-1585.

KALLICH, J.D, N.S. TCHEKMEDYIAN, A.M. DAMIANO, J. SHI, T.J. BLACK et M.H. ERDER (2002). «Psychological outcomes associated with anemia-related fatigue in cancer patients », Oncology, vol. 16, n 9, p. 117-124.

KIMBERLY, L., M.A. PARGEON et J. HAILEY (1999). "Barriers to effective cancer pain management: A review of the literature», Journal of Pain and Symptom Management, vol. $18, n^{\circ} 5$, p. $32-42$.

LEYDON, G., M. BOULTON, C. MOYNIHAN, A. JONES, J. MOSSMAN, M. BOUDIONI et K. MCPHERSON (2000). "Cancer patients' information needs and information seeking behavior: In depth interview study », British Medical Journal, no 320, p. 909-913.

MILLS, M.E. et K. SULLIVAN (1999). «The importance of information giving for patients newly diagnosed with cancer: A review of the literature », Journal of Clinical Nursing, vol. 8, n 6 , p. 631-642.

MORROW, G.R., P.L. ANDREWS, J. HICKOK, J.A. ROSCOE et S. MATTESON (2001). "Fatigue associated with cancer and its treatment», article présenté dans le cadre du Symposium Supportive Care in Cancer à Copenhague, Danemark.

PAILLÉ, P. (1994). L'analyse par théorisation ancrée, Cahiers de recherche sociologique, $\mathrm{n}^{\circ} 23$, p. 147-181.

PAILLÉ, P. et A. MUCCHIELLI (2003). L'analyse qualitative dans les sciences humaines et sociales, Paris, Armand Colin.

RICHER, M.C. et H. EZER (2002). «Living in it, living with it, and moving on: Dimension of meaning during chemotherapy", Oncology Nursing Forum, vol. 29, n ${ }^{\circ}$ 1, p. 113-119.

SAILLANT, F. (1988). Cancer et culture. Produire le sens de la maladie, Montréal, Éditions Saint-Martin.
SILVESTRI G., R. PRITCHARD et H. WELCH (1998). "Preferences for chemotherapy in patients with advanced non-small cell lung cancer: Descriptive study based on scripted interviews », British Medical Journal, n ${ }^{\circ}$ 317, p. 771-775.

SIMES, R.J et A.S. COATS (2001). «Patient preferences for adjuvant of early breast cancer: How much benefit is needed?", Journal of the National Cancer Institute, $\mathrm{n}^{\circ} 30$, p. $146-152$.

SLEVIN, M.L., L. STUBBE, H.J. PLANT et al. (1990). "Attitudes to chemotherapy: Comparing views of patients with cancer with those of doctors, nurses and general public», British Medical Journal, $\mathrm{n}^{\circ} 300$, p. 1458-1460.

STRAUSS, A. et J. CORBIN (1990). Basics of Qualitative Research. Grounded Theory. Procedures and Techniques, Newbury Park, Sage Publications, 263 p.

THE, A.M, T. HACK, G. KOETER et G. VAN DER WAL (2000). «Collusion in doctorpatient communication about imminent death: An ethnographic study", British Medical Journal, no 321, p. 1376-1381.

UITTERHOEVE, R., E. DUIJNHOUWER, B.AMBAUM et T. ACHTERBERG (2003). "Turning toward the psychosocial domain of oncology nursing: A main analysis in the Netherlands », Cancer Nursing, n 26, p. 18-27.

WARD, S.E, K. CARLSON-DAKES, S.H. HUGUES, K.L. KWEKKEBOOM et H.S. DONOVAN (1998). «The impact on quality of life of patient-related barriers to pain management», Research Nursing Health, $\mathrm{n}^{\circ} 21$, p. 405-413.

WARD, S.E, N. GOLDBERG, V. MILLERMCCAULEY et al. (1993). "Patient-related barriers to management of cancer pain », Pain, $\mathrm{n}^{\circ}$ 52, p. 319-324.

WEISS, S.C., L.L. EMANUEL, D.L. FAIRCLOUGH et E.J. EMANUEL (2001). "Understanding the experience of pain in terminally ill patients ", The Lancet, vol. 357, p. 13111315.

WILLIAMS, J., C. WOOD, P. CUNNINGHAMWARBURTON (1999). "A narrative study of chemotherapy-induced alopecia », Oncology Nursing Forum, n 26, p. 1463-1468.

\section{Notes}

1. Cette étude a bénéficié d'une subvention de recherche octroyée par les Instituts de recherche en santé du Canada.

2. Afin de préserver l'anonymat des sujets rencontrés, tous les prénoms utilisés dans les verbatims sont fictifs.

3. Les auteurs remercient Mme Camille Allaire, étudiante en études littéraires, pour ses judicieux conseils quant à la rédaction de cet article. Ils remercient également Mme Andréa Monette, étudiante en travail social, pour son aide quant à la recension des écrits. 